\title{
Novel polygenetic variants evidenced in a patient with Jervell and Lange-Nielsen syndrome
}

\author{
Ana Cecilia Cepeda-Nieto ${ }^{1}{ }^{\circledR}$, Carlos Ramiro Zamora-Alemán $^{1}{ }^{\circledR}$, \\ Mauricio Cortes-Aguirre ${ }^{1}$, Roberto Valdés-Charles ${ }^{1}$, Cesar Rojas-Sánchez ${ }^{1}$, \\ Mauricio Andrés Salinas-Santander ${ }^{1}\left[\right.$, Dan $\mathrm{Hu}^{2}{ }^{\circledR}$, Hector Barajas-Martinez ${ }^{3}$ (]) \\ ${ }^{1}$ Departamento de Investigacion, Facultad de Medicina Unidad Saltillo, \\ Universidad Autónoma de Coahuila, Saltillo, Coahuila, Mexico \\ ${ }^{2}$ Department of Cardiology and Cardiovascular Research Institute, \\ Renmin Hospital of Wuhan University, Wuhan, China \\ ${ }^{3}$ Department of Cardiovascular Research, Lankenau Institute for Medical Research, \\ Wynnewood, PA, United States
}

Jervell and Lange-Nielsen syndrome (JLNS) is an ion channel-caused cardioauditory syndrome characterized by a congenital neurosensorial bilateral deafness and a long QT interval. JLNS is inherited in an autosomal recessive manner and is caused by mutations in the $K C N Q 1$ gene (potassium voltage-gated channel subfamily $Q$ member 1) [1].

The proband was an 8-year-old male who presented with a family history of sudden death and congenital sensorineural deafness. Initial clinical evaluation of the proband showed a mild cranial trauma and minor occipital subgaleal hematoma. No significant cardiovascular findings were noted, but electrocardiography (ECG) analysis (ECG Edan SE-1200, USA) revealed a prolonged QT/QTc in the lead II (420/460 ms) (Fig. 1A, left). Despite the use of beta-blocker $(2 \mathrm{mg} / \mathrm{kg} /$ day $)$ therapy at home, the patient experienced a syncopal event related to emotional stress.

Viskin test [2] was performed, and the results did not support an long QT syndrome (LQTS)-related orthostatic event (baseline QTc $465 \mathrm{~ms}$ in resting phase, QTc MHR of $492 \mathrm{~ms}$, and QTc recovery of $444 \mathrm{~ms}$ ). An adrenaline test [3] was performed at doses of $0.025 \mu \mathrm{g} / \mathrm{kg} / \mathrm{min}$, enabling a QTc of 550 ms without an arrhytmic event
(Fig. 1A, right), and met LQTS electrocardiographic criteria. QTc $686 \mathrm{~ms}$ was observed under stress after placement of the implantable cardioverterdefibrillator (ICD) and beta-blockers. At follow up, the patient was ventricular arrhytmia-free or shock therapy during the 4 years after ICD implantation. After clinical and cardiac electrophysiology, the patient was diagnosed with JLNS.

Molecular genetic analysis was performed by next generation sequencing in the proband, and 4 family members were clinically affected (Fig. 1B). Electrocardiographic assessment of the mother and maternal grandparents revealed borderline QTc values. The electrocardiographies were measured by the Bazett formula. Only the index case was genotyped and the family members declined to do genetic testing until there was more evidence that genetic testing had to be recommended by a genetic counselor.

High throughput DNA sequencing was performed using an Ion Torrent Personal Genome Machine to target and sequence 87 candidate genes linked with inhereted cardiac arrhythmia syndromes. These candidate genes were selected based on their relative expression in the human heart and their ability to modulate ion channel

Address for correspondence: Hector Barajas-Martinez, PhD, FHRS, Cardiovascular Research Department, Lankenau Institute for Medical Research, Wynnewood, PA 19096, United States, tel: 484-476-8134, fax: 484-476-8533, e-mail: barajasmartinezh@mlhs.org

Received: 2.12.2020 Accepted: 8.06.2021 Early publication date: 16.06.2021

This article is available in open access under Creative Common Attribution-Non-Commercial-No Derivatives 4.0 International (CC BY-NC-ND 4.0) license, allowing to download articles and share them with others as long as they credit the authors and the publisher, but without permission to change them in any way or use them commercially. 
A

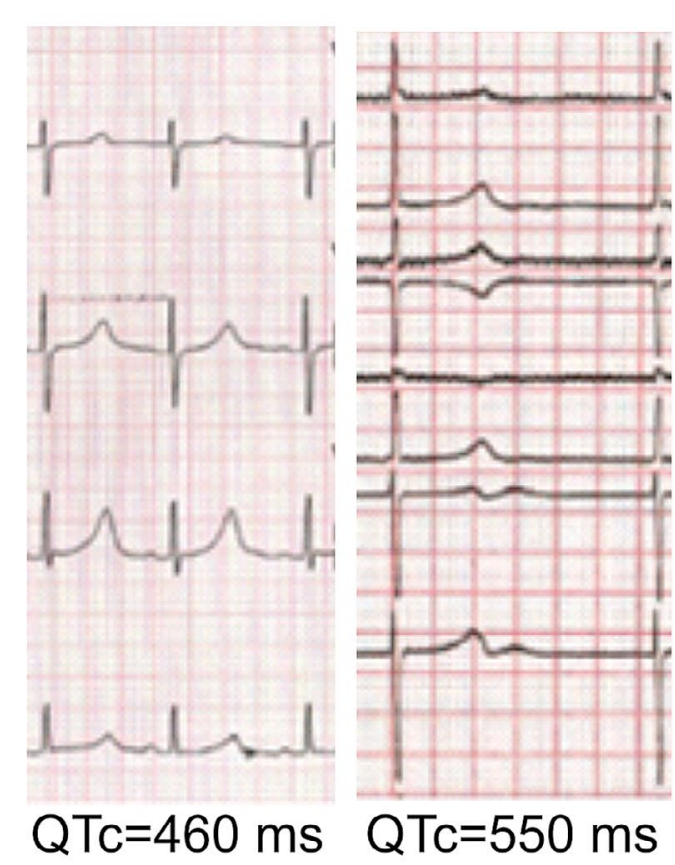

B

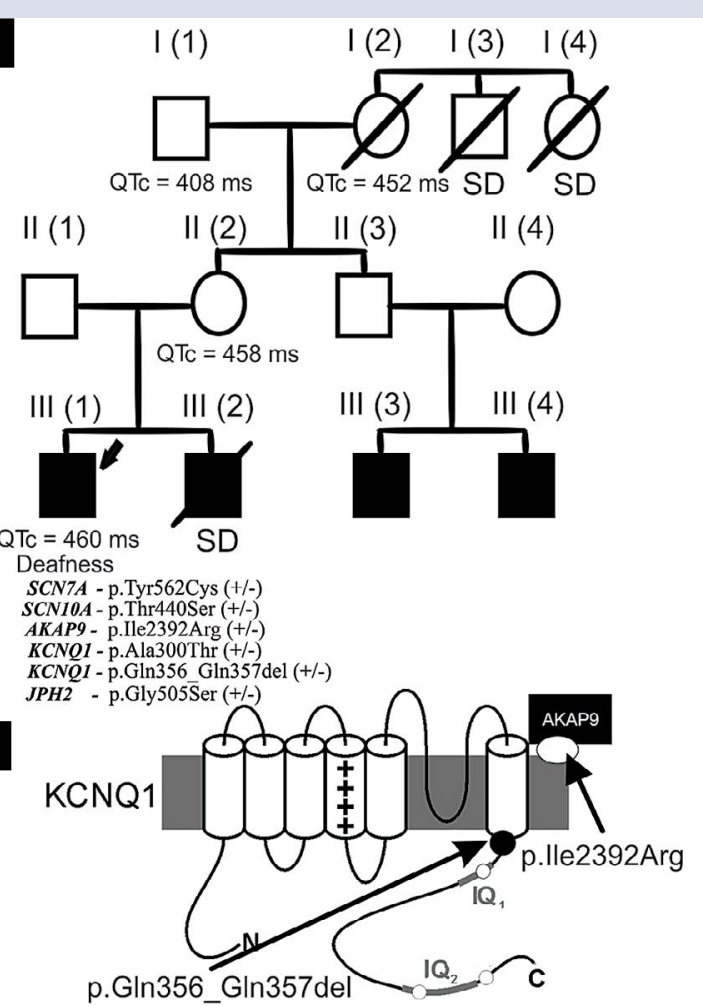

\begin{tabular}{lllllll}
\hline Gene & SCN7A & SCN10A & AKAP9 & KCNQ1 & KCNQ1 & JPH2 \\
\hline Transcript & NM_002976.3 & NM_006514.2 & NM_005751.4 & NM_000218.2 & NM_000218.2 & NM_020433.4 \\
Location & Exonic & Exonic & Exonic & Exonic & Exonic & Exonic \\
Function & Missense & Missense & Missense & Missense & Nonframeshift Deletion & Missense \\
Codon & TGT & TCC & AGA & ACA & - & AGC \\
Exon & 13 & 10 & 31 & 6 & 8 & 4 \\
Protein & p.Tyr562Cys & p.Thr440Ser & p.lle2392Arg & p.Ala300Thr & p.Gln356_Gln357del & p.Gly505Ser \\
Coding & c.1685A>G & c.1318A>T & c.7175T>G & c.898G>A & c.1065_1070delGCAGCA & c.1513G>A \\
Glob MAF & 0.01 & 0.00 & 0.00 & 0.00 & 0.00 & 0.013 \\
MXLL MAF & 0.0234 & 0.00 & 0.00 & 0.00 & 0.00 & 0.0078 \\
SIFT & Tolerated & Tolerated & Damaging & Damaging & Damaging & Tolerated \\
Polyphen & Probably Damaging & Benign & Benign & Probably Damaging & Damaging & Benign \\
Grantham & Radical & Conservative & Non Conservative & Conservative & Conservative & Conservative \\
OMIM & 182392 & 604427 & 604001 & 607542 & 607542 & 605267 \\
\hline
\end{tabular}

$S C N 7 A$, Sodium Voltage-Gated Channel Alpha Subunit 7; SCN10A, Sodium Voltage-Gated Channel Alpha Subunit 10;AKAP9, A-Kinase Anchoring Protein 9. $K C N Q 1$, Potassium Voltage -Gated Channel Subfamily Q Member; JPH2, Junctophilin 2; Global MAF, Global minor allele frequency;

MXL MAF, Mexicans minor allele frequency; SIFT, Sorting Intolerant From Tolerant; OMIM, Online Mendelian Inheritance in Man.

Figure 1. A. Clinical electrocardiography analysis; Left. Electrocardiography during the first syncopal event with QTc of 460 ms by Bazett formula; Right. Electrocardiography during the adrenaline test with QTc 550 ms by Bazett formula at the second syncopal event; B. Patient's pedigree; I (1). Maternal grandfather; I (2). Maternal grandmother; I (3). Maternal granduncle; I (4). Maternal grandaunt; II (1). Patient's father; II (2). Patient's mother; II (3). Maternal uncle; III (1). Patient; III (2). Patient's brother; III (3). Patient's cousin; III (4). Patient's cousin; SD — sudden death; C. Schematic molecular genetics and localization of KCNQ1 mutation and AKAP9 novel genetic variant. Long QT syndrome, like severe Jervell and Lange-Nielsen syndrome, is associated with sudden cardiac death syndromes. $K C N Q 1$ mutation and $A K A P 9$ variant and proteins location and possible interaction in the C-Terminus in the plasma membrane that maybe expressed in the brain, muscle and heart tissues; D. Molecular genetics of novel genetic variants (AKAP9; JPH2; SCN10A; SCN7A) and KCNQ1 mutations using bioinformatics. 
expression and function: $A B C C 8, A B C C 9, A C T C 1$, ACTN2, AGTR1, AKAP9, ANK2, CACNA1C, CACNA2D1, CACNA2D2, CACNB1, CACNB2, CACNB3, CACNB4, CACNG4, CACNG5, CACNG6, CALM1, CALM2, CASQ2, CAV1, CAV2, CAV3, DSG2, DSP, DPP6, DPP7, DPP8, DPP9, DPP10, FGF12, FGF13, GATAD1, GJA5, GLA, GPD1L, HCN2, HCN4, HEY2, IRX3, IRX4, IRX5, JPH2, KCNA4, KCNA5, KCNN1, KCNN2, KCNN3, KCNK1, KCNK2, KCNK3, KCND3, KCNE1, KCNE2, KCNE3, KCNE4, KCNE5, KCNH2, KCNIP2, KCNJ2, KCNJ8, KCNJ9, KCNJ10, KCNQ1, KCNQ2, PKP2, PRKAG2, PXDNL, RYR2, SCN1A, $S C N 2 A, S C N 3 A, S C N 4 A, S C N 5 A, S C N 7 A, S C$ N10A, SCN1B, SCN2B, SCN3B, SCN4B, SEMA3C, SNTA1, SUR1A, SUR2A, TBX5, TRPM4, TTN. All genetic variants uncovered were confirmed using the gold standard Sanger sequencing. Polymerase chain reaction $(\mathrm{PCR})$ products were purified with a commercial enzyme (ExoSAP- IT, USB, Cleveland, $\mathrm{OH}$ ) and directly sequenced from both directions using BigDye Terminator v3.1 chemistry on an Applied Biosystems 3730DNA Analyzer (Life Technologies, Carlsbad, CA).

After signal processing and basecalling, the Ion Torrent Suite software was used to map the sequencing reads to the DNA reference sequence (hg19) and identify variants through the Variant Caller plugin as well as the Ion Reporter analysis tool. Ion Reporter compares all variations identified against NCBI's dbSNP to rule out common SNPs, as well as the 1000 Genomes Project and Exome Sequencing Project to get published frequencies. All variations uncovered were probed in $200-400$ healthy ethnically matched controls. Genetic variant under $0.05 \%$ minor allele frequency were considered mutations and above $0.05-2.5 \%$ rare variants, following American College of Medical Genetics (ACMG) recommendations [4]. All variants were analyzed using several pathogenicity in silico prediction tools such as PolyPhen-2, SIFT and Grantham.

Three novel heterozygous exonic were identified, likely benign variants to be associated in this index patient diagnosed with JLNS, AKAP9(p.Ile2392Arg); JPH2(p.Gly52Ser); SCN10A(p.Thr440Ser), and one moderate pathogenic rare variant $S C N 7 A$ (p.Tyr562Cys) [4]. Two mutations in $K C N Q 1$ were already discovered in the patient (p.Gln356_Gln357del and p.Ala300Thr) [5, 6]. According to available research, this is the first evidence of polygenic variants in a confirmed case of clinically severe JLNS. In this case, polygenic variants may be explained by consanguineous relations among the patient's relatives, consistent with local traditions still prevalent in small populations. Surprisingly, the ECG abnormalities manifested only in the index patient who carried the mutations and genetic variants in five different genes with a very interesting double deletion in $K C N Q 1$ with a close physical protein-protein interaction with the AKAP9 gene (Fig. 1C). Based on the clinical and ECGs phenotype associated with LQTS one of the main culprit genes could be the doble mutations in the KCNQ1 gene and genetic variant in AKAP9 to induce QT prolongation. It has been described that multiple genes identified could play together a role in the development of the LQTS phenotype at the same time or to be associated with any cardiac arrhythmia syndrome [6].

Genetic variants found in the case reported have been rarely associated with disease in previous reports. The observed variant $J P H 2$ (p.Gly505Ser) has been related to hypertrophic cardiomyopathy [7], whereas the relationship of the observed variants in SCN1OA and SCN7A genes have not been previously characterized for a JLNS-related phenotype. In another context, almost 30 pathogenic genetic variants of $K C N Q 1$ gene have been associated with JLNS [8]; meanwhile, both deletion and duplication of one or more exons of $K C N Q 1$ are known to cause LQTS [9]. In the present study the two mutations in $K C N Q 1$ have been related with LQTS and sudden unexpected death syndromes $[5,6] . K C N Q 1$ also has been found to co-interact with $A K A P 9$ by reducing the IKs channels, and it has been associated with prolongation of the QT interval as a potential marker for long QT type 1-modified effects [10].

Localization of the KCNQ1 mutations and AKAP9 genetic variant in the proband are shown in Figure 1D. The hypothesis herein, is based on the possible loss-of-function in the potassium in comparison to WT channels when predicted by in silico prediction [11]; however, in vitro functional studies may need it to clarify the ionic mechanisms. These potential pathophysiological deficiencies may alter the phenotypic manifestation of LQTS as well as the responsiveness to pharmacological therapies.

In summary, four novel genetic variants were found [AKAP9(p.Ile2392Arg); JPH2(p.Gly52Ser); $S C N 10 A$ (p.Thr440Ser) and SCN7A(p.Tyr562Cys)] and two known mutations in $K C N Q 1$ in a patient with ventricular arrhythmias with similarities to long QT type 1-modified effects. Moreover, none of these variants has been linked to either LQTS or other sudden cardiac death syndromes.

Conflict of interest: None declared 


\section{References}

1. Schulze-Bahr E, Wang Q, Wedekind H, et al. KCNE1 mutations cause jervell and Lange-Nielsen syndrome. Nat Genet. 1997; 17(3): 267-268, doi: 10.1038/ng1197-267, indexed in Pubmed: 9354783.

2. Mazzanti A, Priori SG. Diagnosis of long QT syndrome: time to stand up! Rev Esp Cardiol (Engl Ed). 2017; 70(11): 898-900, doi: 10.1016/j.rec.2017.05.004, indexed in Pubmed: 28602389.

3. Clur SAB, Chockalingam P, Filippini LH, et al. The role of the epinephrine test in the diagnosis and management of children suspected of having congenital long QT syndrome. Pediatr Cardiol. 2010; 31(4): 462-468, doi: 10.1007/s00246-009-9603-2, indexed in Pubmed: 19957170.

4. Richards S, Aziz N, Bale S, et al. ACMG Laboratory Quality Assurance Committee. Standards and guidelines for the interpretation of sequence variants: a joint consensus recommendation of the American College of Medical Genetics and Genomics and the Association for Molecular Pathology. Genet Med. 2015; 17(5): 405-424, doi: 10.1038/gim.2015.30, indexed in Pubmed: 25741868

5. Antúnez-Argüelles E, Rojo-Domínguez A, Arregui-Mena AL, et al. Compound heterozygous KCNQ1 mutations (A300T/P535T) in a child with sudden unexplained death: Insights into possible molecular mechanisms based on protein modeling. Gene. 2017; 627: 40-48, doi: 10.1016/j.gene.2017.06.011, indexed in Pubmed: 28600177.
6. Napolitano C, Priori SG, Schwartz PJ, et al. Genetic testing in the long QT syndrome: development and validation of an efficient approach to genotyping in clinical practice. JAMA. 2005; 294(23): 2975-2980, doi: 10.1001/jama.294.23.2975, indexed in Pubmed: 16414944

7. Matsushita Y, Furukawa T, Kasanuki H, et al. Mutation of junctophilin type 2 associated with hypertrophic cardiomyopathy. J Hum Genet. 2007; 52(6): 543-548, doi: 10.1007/s10038-0070149-y, indexed in Pubmed: 17476457.

8. Piippo K, Swan H, Pasternack M, et al. A founder mutation of the potassium channel KCNQ1 in long QT syndrome: implications for estimation of disease prevalence and molecular diagnostics. J Am Coll Cardiol. 2001; 37(2): 562-568, doi: 10.1016/s07351097(00)01124-4, indexed in Pubmed: 11216980.

9. Eddy CA, MacCormick JM, Chung SK, et al. Identification of large gene deletions and duplications in KCNQ1 and $\mathrm{KCNH} 2$ in patients with long QT syndrome. Heart Rhythm. 2008; 5(9): 1275-1281, doi: 10.1016/j.hrthm.2008.05.033, indexed in Pubmed: 18774102

10. Chen L, Marquardt ML, Tester DJ, et al. Mutation of an A-kinase-anchoring protein causes long-QT syndrome. Proc Natl Acad Sci USA. 2007; 104(52): 20990-20995, doi: 10.1073/ pnas.0710527105, indexed in Pubmed: 18093912.

11. Nof E, Barajas-Martinez H, Eldar M, et al. LQT5 masquerading as LQT2: a dominant negative effect of KCNE1-D85N rare polymorphism on KCNH2 current. Europace. 2011; 13(10): 1478-1483, doi: 10.1093/europace/eur184, indexed in Pubmed: 21712262. 